ANNALES

UNIVERSITATIS MARIAE CURIE-SKŁODOWSKA

LUBLIN - POLONIA

VOL. LXX, 1

SECTIO AA

2015

\title{
On mechanism of the bubble bouncing from hydrophilic and hydrophobic solid surfaces
}

\author{
Jan Zawała ${ }^{*}$, Piotr Zawała and Kazimierz Małysa \\ Jerzy Haber Institute of Catalysis and Surface Chemistry PAS, \\ ul. Niezapominajek 8, Kraków \\ *e-mail: nczawala@)cyf-kr.edu.pl
}

The kinetics of collision and bouncing of an air bubble on hydrophilic and hydrophobic solid surfaces immersed in distilled water is reported. We carried out the experiments and compared the bubble collision and bouncing courses on the stagnant and vibrating, with a controlled frequency and amplitude, solid/liquid interface. For stagnant interface differences in the outcome of the bubble collisions with hydrophilic and hydrophobic solid surfaces are resulting from different stability of the intervening liquid film formed between the colliding bubble and these surfaces. The liquid film was unstable at Teflon surface, where the three-phase contact (TPC) and the bubble attachment were observed, after dissipation of most of the kinetic energy associated with the bubble motion. For vibrated solid surface it was shown that kinetics of the bubble bouncing is independent on the hydrophilic/hydrophobic properties of the surface. Similarly like at water/glass hydrophilic interface, even at highly hydrophobic Teflon surface time of the bubble collisions and bouncing was prolonged almost indefinitely. This was due to the fact that the energy dissipated during the collision was re-supplied via interface vibrations with a properly adjusted acceleration. The analysis of the bubble deformation degree showed that this effect is related to a constant bubble deformation, which determined constant radius of the liquid film, large enough to prevent the draining liquid film from reaching the critical thickness of rupture at the moment of collision. The results obtained prove that mechanism of the bubble bouncing from various interfaces 
depends on interrelation between rates of two simultaneously going processes: (i) exchange between kinetic and surface energies of the system and (ii) drainage of the liquid film separating the interacting interfaces.

\section{INTRODUCTION}

Kinetics and outcome of the bubble collision with solid surfaces depends on many interrelated factors, such as: (i) bubble size, impact velocity and degree of shape deformation, (ii) solid surface topography (roughness), hydrophobic/hydrophilic properties and electrical charge, (iii) properties of the liquid film formed by the colliding bubble, etc. Outcome of the bubble collisions, that is, the probability of rebound and/or attachment are factors of significant importance in many industrial processes involving multiphase flows. For example, in flotation process, the collision and attachment of the bubble to solid surface is a "heart" of separation phenomenon $[1,2]$. Rupture of the liquid film, separating surfaces of the colliding grains and bubbles, and formation of stable bubble-grain aggregate is the elementary process of a crucial importance for effective recovery of useful minerals. The bubble-grain aggregate can be formed only during mutual contact of the liquid/gas and liquid/solid interfaces, which timescale is generally of an order of a few milliseconds only. Therefore, for increased attachment efficiency the contact time has o be prolonged to reduce probability of the bubble rebound. Knowledge about the mechanism of bubble collision, bouncing and attachment processes is needed for better understanding of problems involving the role of the bubble collision parameters in kinetics of the three-phase contact (gas/liquid/solid - TPC) formation.

It is rather generally accepted that there is no the TPC formation at hydrophilic solid surfaces and for rapid bubble rupture and the TPC formation, the solid surface has to be hydrophobic [3, 4]. It was recently shown, however, that even at the solid/liquid interfaces of high hydrophobicity (contact angle above $100^{\circ}$ ) immersed in distilled water the duration of the TPC formation, i.e., the total time needed for bubble attachment to solid surface, can vary by over order of magnitude [5-7]. The bubble could be either attached to the solid surface immediately during the first collision or four to five bouncing periods were observed before the bubble attachment. This difference was attributed to different stability and kinetics of drainage of separating liquid film formed between 
the colliding bubble and solid/liquid interface. Indeed, kinetics of the liquid film drainage is a parameter of crucial importance for the collision outcome. During the collision, the formed liquid film needs to drain to the thickness, which ensures the film rupture and TPC hole formation. This is so-called a critical thickness of rupture of the film.

Generally, as was theoretically postulated by Chesters and Hofman [8] for the bubble collisions with liquid/gas interface, the kinetic energy associated with the bubble motion is transferred, during the bubble collision and liquid film drainage, into the bubble potential energy (surface energy related to enlarged the bubble surface area during the collision). The bubble bounces only if the transfer between these two energy components is faster than the film drainage to its critical rupture thickness. Otherwise the bubble ruptures at surface of a pure liquid. The probability of bounce is therefore related to interrelation between rate of energy transfer and kinetics of liquid film drainage. This theoretically postulated mechanism had been confirmed later on the basis of experimental observations [9] and numerical calculations [10,11]. However, no direct experimental evidence was presented in the literature. The direct experimental confirmation of this mechanism correctness was published quite recently for the bubble colliding with pure silicone oil [12] and distilled water [13] surfaces. It was shown that the bubble bouncing at these free surfaces could be prolonged almost indefinitely when the kinetic energy is resupplied to the system from the external source. This was done using novel experimental technique [12, 13], where the bubble collided with liquid/gas interface being vibrated with precisely controlled frequency and amplitude. At vibrated water/air interface the bubble lifetime was prolonged by dozens orders of magnitude, and the phenomenon of formation of "immortal" bubbles were observed [13].

In this paper we show that prolongation of the bubble bouncing can be obtained even in the case of hydrophobic solid surface immersed in distilled water. Applying the similar experimental methodology, described in [13], it was demonstrated that also in the case of the bubble collisions with solid surfaces (hydrophilic and hydrophobic) the bubble bouncing could be prolonged indefinitely. The results obtained show that even in the case of highly hydrophobic solid surfaces the time of the TPC formation and the bubble attachment can be prolonged significantly if the energy dissipated during the bubble collisions is re-supplied. Moreover, the data obtained document correctness and a general character of the bubble bouncing mechanism described above. 


\section{MATERIALS AND METHODS}

The experimental set-up used to study influence of solid/liquid interface vibrations on bubble collision, bouncing and kinetics of the TPC formation is presented schematically in Fig. 1. The set-up consists of: (i) square glass column filled with distilled water, (ii) glass capillary (inner diameter of $0.075 \mathrm{~mm}$ ) sealed at the column bottom, (iii) highspeed camera, (iv) electromagnetic shaker connected to the electronic devices allowing control of frequency and amplitude of vibrations, and (v) gas-tight syringe connected with syringe pump. The hydrophobic smooth Teflon plate (contact angle $\sim 100^{\circ}$ ) or hydrophilic glass plate was attached to the shaker moving table and immersed in distilled water at the distance ca. 7-8 cm above the capillary orifice. This distance was enough for the bubble to reach its terminal velocity $(\mathrm{u}=34.7 \mathrm{~cm} / \mathrm{s})$ before the collision. The Teflon plate was polished before the experiments using a sand paper of grid number 2500. Depending on the desired experimental conditions, the Teflon plate was at rest (shaker was turned off) or was vibrating with precisely controlled acceleration (frequency and amplitude). The equivalent diameter of the bubble $\left(d_{e q}\right)$ detaching from the capillary was $1.48 \pm 0.03 \mathrm{~mm}$. A high-speed camera (1000 frames per second) was used to monitor dynamic phenomena occurring during the

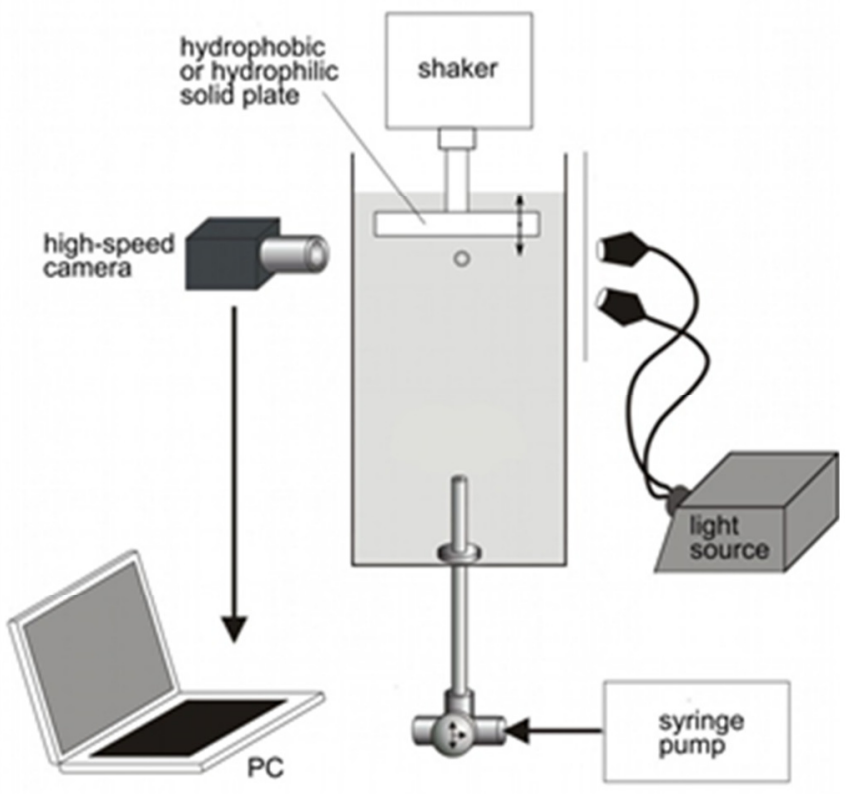

Fig. 1. Scheme of the experimental set-up. bubble collision with solid surface.

The recorded movies were analyzed using image analysis software to determine spatial displacement of the bubble geometrical center and its local velocity. Further details of the experimental set-up and determination of the bubble velocity have been described elsewhere [7,13]. Prior to every experiment the glass 
elements of the set-up as well as Teflon and glass plates were washed with Mucasol, commercial cleaning liquid, and then rinsed with large quantity of distilled water. The experiments were carried out in room temperature $\left(20-22^{\circ} \mathrm{C}\right)$.

\section{RESULTS AND DISCUSSION}

\subsection{Solid/liquid interfaces at rest}

Sequences of photos illustrating the bubble collision with hydrophilic glass and hydrophobic smooth Teflon plates are shown in Fig. 2. The time interval between successive bubble positions is $1 \mathrm{~ms}$. The moment of the first bubble collision was arbitrary denoted as $t=0$. The respective time of beginning of each collision sequence (rows in Fig. 2) are marked at the first image of the sequence. The bubble approaches the solid surfaces at terminal velocity and a constant degree of deformation. As can be observed, after a short moment of bubble-solid surface contact, the bubble bounces backward, i.e. starts to move in the direction opposite to the buoyancy force, with rapid shape pulsations. After energy dissipation the bubble backward motion is stopped and second approach to the surface begins. Consequently, a second collision is observed and the entire sequence (approach and bounce) is repeated. As seen in Fig. 2, as a consequence of energy dissipation, the amplitude of each successive bounce decreases, resulting in reduction of the bubble deformation degree. The approach-bounce cycles with diminishing amplitude can be observed until the energy associated with bubble motion is completely dissipated, i.e. the bubble stays in permanent contact with the solid surface (see last row of the sequence). Note that until the bubble kinetic energy is almost completely dissipated the courses of the bubble collisions with the glass and Teflon plates are identical. This means that they are not dependent on the solid hydrophobic/hydrophilic properties. The significant difference in the collision outcome can be observed after most of energy dissipation, i.e. when the bubble stays in contact with the solid plate. As can be seen, in the case of hydrophilic glass surface the bubble is captured motionless beneath the solid/liquid interface, without formation of the TPC. In contrary, at Teflon plate the water film, separating the Teflon and bubble surfaces, ruptures and the TPC is formed. The moment of formation of the TPC hole is marked in the last row of the bottom sequence presented in Fig. 2. This different bubble 
behaviour is a consequence of different stability in the separating liquid film formed between the bubble and solid surface.

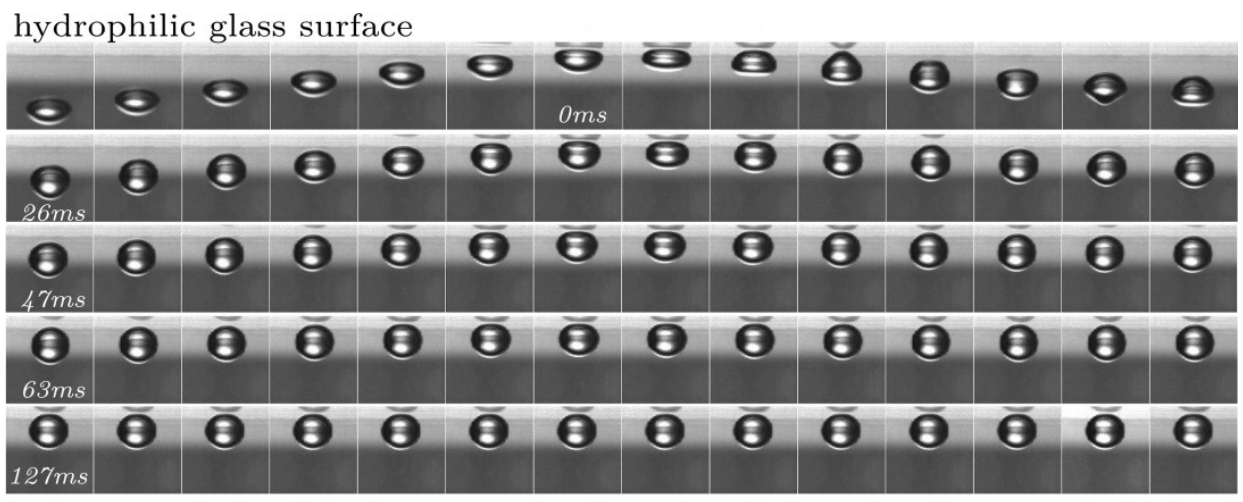

hydrophobic T2500 surface

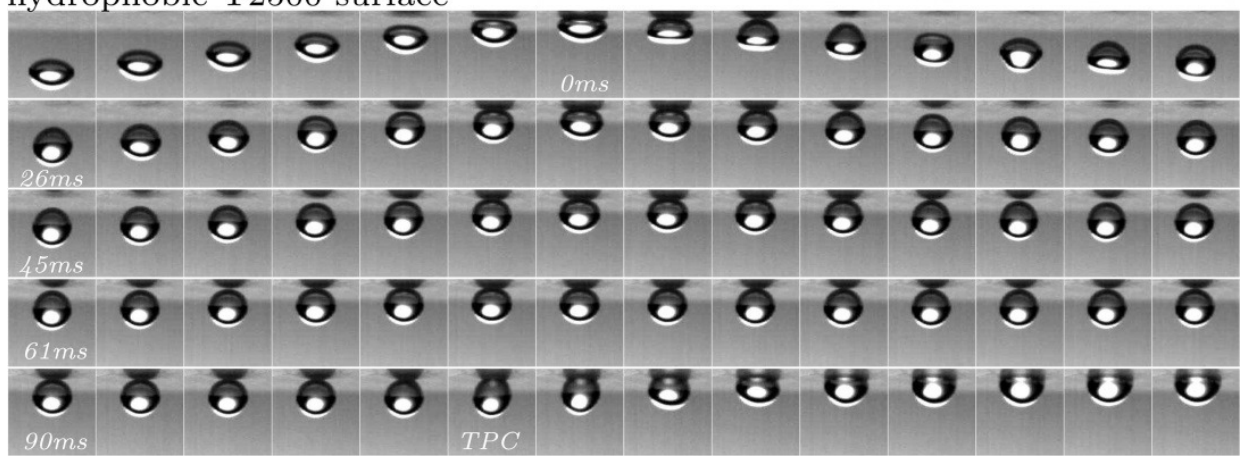

Fig. 2. Sequences of photos showing the bubble collisions and bouncing at hydrophilic glass and smooth hydrophobic Teflon plates.

Variations in position of the bubble geometrical center are presented in Fig. 3, both for the glass and Teflon surfaces. The solid surface position was arbitrarily chosen as $y_{c}=0$. It is clearly seen here that until the water film ruptures and the TPC is formed (rapid jump at $y_{c}=f(t)$ profile seen in Fig. 3), the kinetics of the bubble collisions and bouncing, that is, the amplitude of the bubble rebounds (Fig. 3) and its velocity variations (Fig. 4), are identical, i.e. independent on the solid surface nature. As seen, the time of the TPC formation $\left(t_{T P C}\right)$, i.e. the time span from the moment of the first collision $(t=0)$ to the TPC formation is 96 $\mathrm{ms}$. The average $t_{T P C}$ calculated from 10 independent runs was equal 104 $\pm 18 \mathrm{~ms}$. 


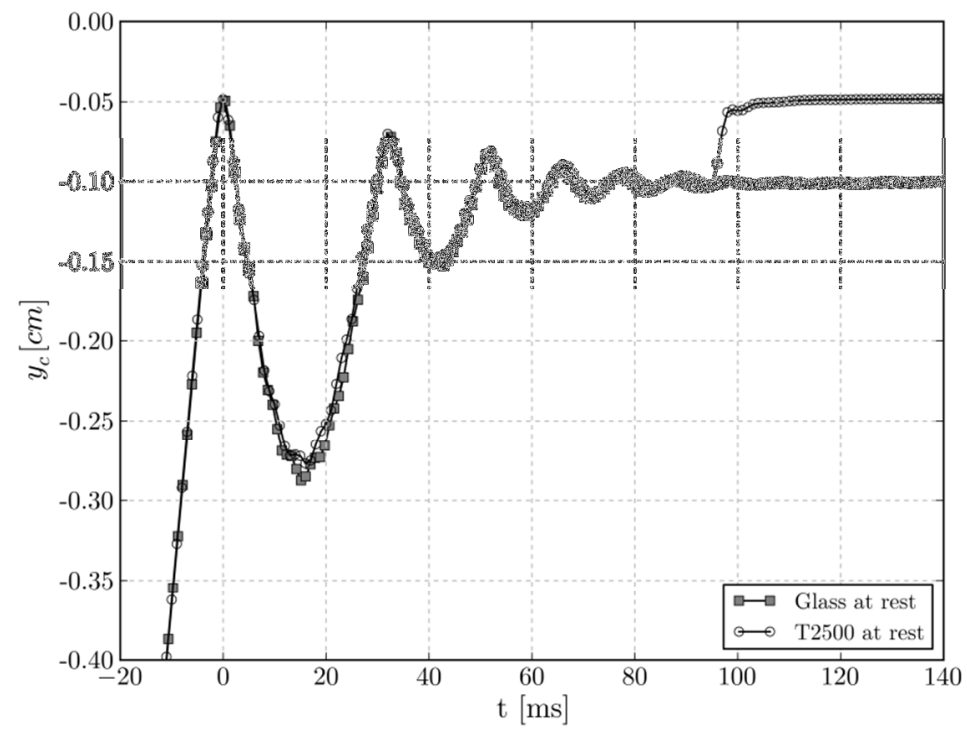

Fig. 3. Variations of the positions of the bubble geometrical center, in respect to the solid/liquid interface, during the bubble bouncing from the glass and Teflon surfaces.

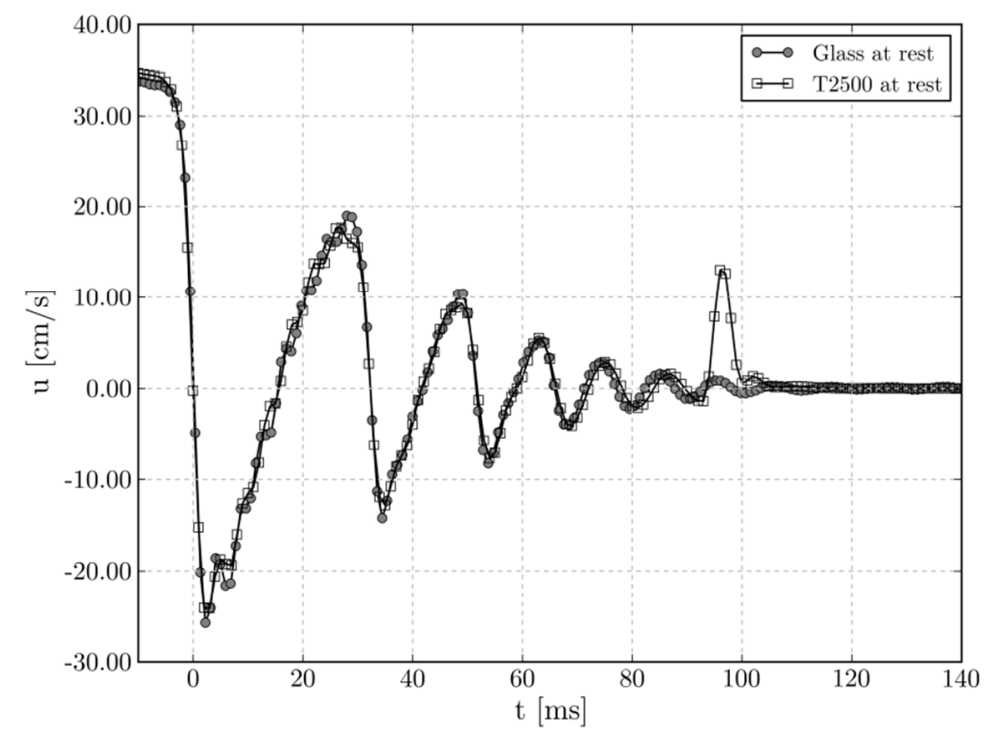

Fig. 4. Variations of the bubble local velocities during collisions with hydrophilic (glass) and hydrophobic (smooth Teflon) solid surfaces.

Similar observations can be made in Fig. 4, where the bubble local velocity variations with time are presented. As seen, the bubble 
approaches to the solid surfaces with identical, terminal velocity equal $34.7 \mathrm{~cm} / \mathrm{s}$. The velocity variations at the Teflon and glass surfaces are the same. The TPC is formed at the Teflon plate, what is associated in sudden jump in the bubble position (see. Fig. 3), and consequently, high peak in velocity variations.

\subsection{Vibrating solid/liquid interfaces}

The variations in the bubble positions during collisions and bouncing at vibrating glass and Teflon surfaces are presented in Fig. 5. The frequency of vibrations of both studied surfaces was $90 \mathrm{~Hz}$ and the acceleration of vibrations was equal $\Gamma=1.39 \mathrm{~g}$, where $g$ is the gravity acceleration. Note please, that the time-scale of the phenomena recording (the range of $x$-axis) is significantly longer here. Similarly, like in the case of the interfaces being in rest, the first collision and initial bounces of the bubble were almost identical for vibrating Teflon and glass surfaces. Here, however, after dissipation of the most of the kinetic energy associated with velocity of the rising bubble, due to periodical vibrations of the solid/liquid interfaces, the bubble is not captured motionless beneath the solid surface. Solid surface vibrations mean that the energy dissipated is constantly resupplied to the system, so the bubble can bounce at the surface almost indefinitely. Moreover, no TPC formation is observed at hydrophobic Teflon surface within the presented time span. The bubble bounces from the hydrophobic Teflon surface with constant amplitude and its attachment to the hydrophobic surface does not occur similarly, as in the case of the hydrophilic glass surface. The only difference is the phase-shift of the bubble oscillation periods (see insert in Fig. 5). This is a consequence of the fact that the oscillating solid surface was in a random position at the moment of the bubble first collision. Careful analysis of the bubble bouncing period (see insert in Fig. 5) revealed that it is equal to $T=11 \mathrm{~ms}$, i.e. the period of the solid plate vibrations $(T=1 / f$, where $f=90 \mathrm{~Hz})$. The results presented in Fig. 5 show that when the kinetic energy, which is being dissipated due to the viscous losses, is resupplied in a proper manner from the external source, the bouncing of the bubble at hydrophobic solid surface without the TPC formation can be significantly (indefinitely) prolonged. The reasons of this spectacular phenomenon are explained below (next paragraph). 


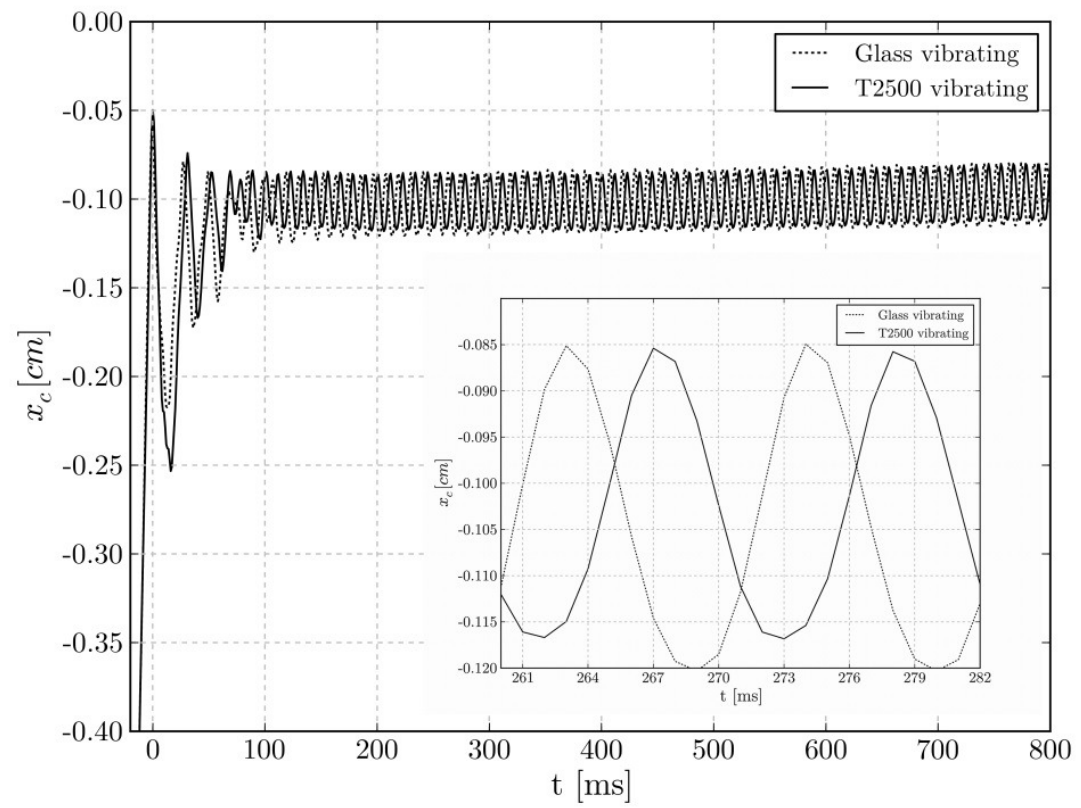

Fig. 5. Variations of the positions of the bubble geometrical center, in respect to the solid/liquid interface, during the bubble bouncing from the vibrating glass and Teflon surfaces $(90 \mathrm{~Hz}, \Gamma=1.39 \mathrm{~g})$.

Figure 6 presents the photos of the bubble at the moment of subsequent collisions with Teflon surface being at rest and vibrating with $f=90 \mathrm{~Hz}$. There are presented photos of the bubbles at the collision moments when the degree of their shape deformation is at the maximum. The collision number is written at each photo. As already discussed above, in the case of stagnant water/solid interface, after the 5th collision the bubble stays in permanent contact with the solid plate and only residual shape pulsations can be observed. Shortly later the TPC is formed at hydrophobic Teflon surface, i.e. the water film reaches its critical thickness of rupture and the bubble is attached to the Teflon surface (see top row photos in Fig. 6). Moreover, the bubble deformation degree diminishes with collision number and the rupture of the bubble takes place, when the deformation is the smallest. This is, however, not the case at vibrating Teflon surface. Here, after initial dissipation of kinetic energy and equalization of the solid/liquid interface and the bubble oscillation periods, the deformation degree of the bubble is practically constant, i.e., it stops to change with the collision number. Moreover, the bubble deformation degree at the vibrating Teflon surface 
is always higher than the deformation degree just before the bubble rupture and the TPC formation at the stagnant Teflon surface.

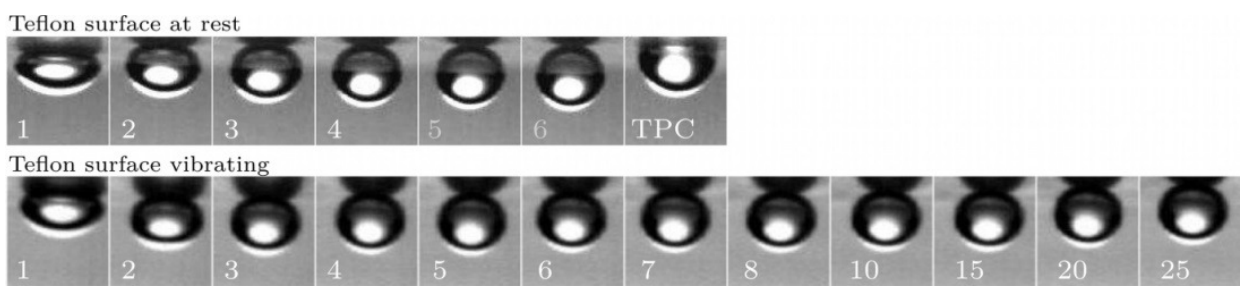

Fig. 6. Photos of the bubble at the moment of its maximum deformation during subsequent collision with stagnant and vibrating $(90 \mathrm{~Hz}, \Gamma=1.39 g)$ Teflon/water interface.

Quantitative data supporting the analysis presented above are shown in Fig. 7. There are presented values of the bubble horizontal diameter at the moment of its maximum deformation during collisions with stagnant and vibrating Teflon surfaces. In the case of the Teflon surface being at rest, the $d_{h}$ decreases with the collision number. This is also the case for first five collisions at the vibrating surface. Here, however, after the short period, the $d_{h}$ start to be constant. It can be clearly seen in Fig. 7 that the value of $d_{h}$ at vibrating surface is always higher than the threshold $d_{h}$ value, for which the TPC is formed at stagnant Teflon surface.

The presented data, showing significant prolongation of the bubble bouncing time at vibrating hydrophobic surface, is the consecutive and strong experimental evidence showing that outcome of the bubble collision with the interface depends on competition between two simultaneous processes: (i) exchange between kinetic and surface energies of the system and (ii) drainage of the liquid film separating the interacting interfaces - here the water/gas and water/solid interfaces. In our opinion the degree of the bubble shape deformation is a crucial parameter, which decides about the relations in timescales of these two simultaneous processes.

As was shown recently [14] the radius of the film formed $\left(R_{f}\right)$ by the bubble colliding with liquid/gas interface can be related to the bubble $d_{h}$ by the following expression:

$$
R_{f}=\sqrt{\frac{E_{k} d_{h}}{2 \pi \sigma_{e q} \Delta d_{v}}}
$$


where $E_{k}$ is kinetic energy associated with the bubble motion, $\sigma_{e q}$ is equilibrium surface tension of liquid and $\Delta d_{v}$ is difference in the bubble vertical diameter just before impact and at the moment of the maximum shape deformation. As seen (Eq. 1), the radius of the liquid film

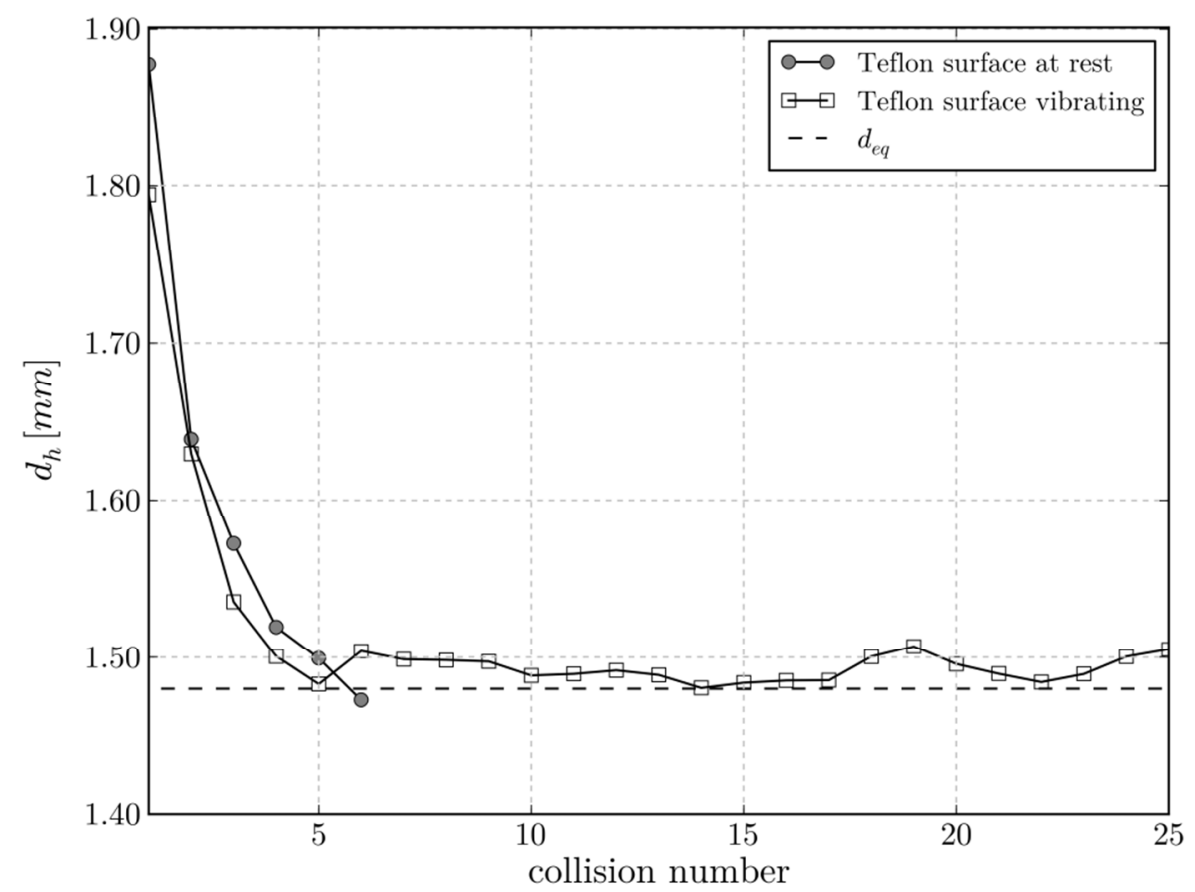

Fig. 7. Values of the bubble maximum horizontal diameter at the moment of collision with stagnant and vibrating Teflon surface.

formed is directly proportional to the bubble horizontal diameter $d_{h}$. Thus, an increase of the bubble deformation degree at the moment of collision causes enlargement of the film radius $R_{f}$, which affects strongly kinetics of the film drainage. For example, according to the simplified equation $[15,16]$ :

$$
-\frac{d h}{d t}=\frac{1}{\left(R_{f}\right)^{n}}
$$

the velocity of the liquid film drainage, i.e. decrease of the film thickness (h) in time $(t)$, is inversely proportional to the $R_{f}$ value at the power of $n$. The $n$ parameter in Eq. 2 depends on the properties of the liquid film interfaces and oscillates in the range $0.8-4.0$ [15-17]. The Eq. 2 shows that the rate of the film drainage velocity decreases always when the film radius $R_{f}$ is larger. Thus, the results presented above (Figs 6 and 7) show 
that the time of the bubbles bouncing at vibrating hydrophobic solid surface was so significantly prolonged as a consequence of persistent enlargement of the size of the water film separating the bubble and vibrating Teflon surface. Larger film size means a prolongation of the film drainage time. Slower drainage means that the time available for the exchange of the kinetic energy, associated with the bubble velocity, into the surface energy can be shorter than the time needed for the liquid film to drain to its critical thickness of rupture.

\section{CONCLUSIONS}

It was shown that time of the bubble collisions and bouncing even at highly hydrophobic solid surface (Teflon) can be prolonged indefinitely when the energy dissipated during the collision is re-supplied via vibrations of the water/Teflon interface with a properly adjusted frequency and amplitude. Thus, in such a manner formation of the threephase contact (TPC) can be prevented or significantly prolonged at highly hydrophobic surfaces. At vibrating hydrophilic glass and hydrophobic Teflon surfaces the kinetic of the bubble bouncing was practically identical. This was a consequence of the constant bubble deformation, which determines constant radius of the liquid film. Due to the liquid/solid vibrations this radius was kept constant and large enough to prevent the draining liquid film from reaching the critical thickness of rupture before exchange of the kinetic energy into the surface energy of the system. The results obtained prove that mechanism of the bubble bouncing from various interfaces consists in competition of the two simultaneously going processes: (i) exchange between kinetic and surface energies of the system and (ii) drainage of the liquid film separating the interacting interfaces - here the water/gas and water/solid interfaces.

\section{ACKNOWLEDGEMENTS}

The study is related to the activity of the COST Action MP1106 "Smart and green interfaces - from single bubbles and drops to industrial, environmental and biomedical applications". Financial support from the Polish National Science Center (NCN grant No. 2013/09/D/ST4/03785) is acknowledged with gratitude. 


\section{REFERENCES}

[1] D. Hewitt, D. Fornasiero, J. Ralston, J. Chem. Soc. Faraday Trans., 91(13), 1997, (1995).

[2] J. Ralston, Bubble-Particle Capture. Encyclopaedia of Separation, (I. D. Wilson Ed.), Academic Press, 2000.

[3] J. Laskowski, J. A. Kitchener, J. Colloid Interface Sci., 29, 670, (1969).

[4] T. D. Blake, J. A. Kitchener, J. Chem. Soc. Faraday Trans., 1, 68 1435 (1972).

[5] M. Krasowska, K. Malysa, Int. J. Miner. Process., 81, 205, (2007).

[6] M. Krasowska, J. Zawala, K. Malysa, Adv. Colloid Interf. Sci., 147-148, 155, (2009).

[7] D. Kosior, J. Zawala, M. Krasowska, K. Malysa, Phys Chem. Chem. Phys., 15, 2586, (2013).

[8] A. K. Chesters, G. Hofmann, Appl. Sci. Res., 38, 353, (1982).

[9] H.-K.Tsao, D. L. Koch, Phys. Fluids, 9(1), 44, (1997).

[10] E. Canot, M. El Hammoumi, D. Lachkar, Theor. Comput. Fluid Dyn., 17, 51, (2003).

[11] J. Zawala, T. Dabros, Phys. Fluids, 25, 12310, (2013).

[12] J. Zawala, S. Dorbolo, D. Terwagne, N. Vandewalle, K. Malysa, Soft Matter, 7, 6719, (2011).

[13] J. Zawala, S. Dorbolo, N. Vandewalle, K. Malysa, Phys. Chem. Chem. Phys., 15(40), 17324, (2013).

[14] J. Zawala, K. Malysa, Langmuir, 27, 2250, (2011).

[15] E. Manev, R. Tsekov and B. Radoev, J. Disp. Sci. Technol., 18, 769, (1997).

[16] D. Exerowa, P. Kruglyakov, Foam and Foam Films, Theory, Experiment, Application, Elsevier, Amsterdam, 1st edn., 1998.

[17] A. Scheludko, Adv. Colloids Interface Sci., 1, 391, (1967). 